\title{
Novel Axillary Approach for Brachial Plexus in Robotic Surgery: A Cadaveric Experiment
}

\author{
Cihangir Tetik ${ }^{1}$ and Metin Uzun ${ }^{2}$ \\ ${ }^{1}$ Department of Hand Surgery, Acıbadem Maslak Hospital, Darüşşafaka Mah. Büyükdere Cad. No. 40, \\ Maslak, Sariyer, Istanbul, Turkey \\ ${ }^{2}$ Orthopaedics and Traumatology Department, Acıbadem Maslak Hospital, Darüşşafaka Mah. Büyükdere Cad. No. 40, \\ Maslak, Sariyer, Istanbul, Turkey
}

Correspondence should be addressed to Metin Uzun; drmetinuzun@gmail.com

Received 15 May 2014; Accepted 8 July 2014; Published 21 July 2014

Academic Editor: Stephen Kavic

Copyright (C) 2014 C. Tetik and M. Uzun. This is an open access article distributed under the Creative Commons Attribution License, which permits unrestricted use, distribution, and reproduction in any medium, provided the original work is properly cited.

\begin{abstract}
Brachial plexus surgery using the da Vinci surgical robot is a new procedure. Although the supraclavicular approach is a well known described and used procedure for robotic surgery, axillary approach was unknown for brachial plexus surgery. A cadaveric study was planned to evaluate the robotic axillary approach for brachial plexus surgery. Our results showed that robotic surgery is a very useful method and should be used routinely for brachial plexus surgery and particularly for thoracic outlet syndrome. However, we emphasize that new instruments should be designed and further studies are needed to evaluate in vivo results.
\end{abstract}

\section{Introduction}

Brachial plexus surgery using the da Vinci surgical robot is a new procedure [1].

To evaluate the advantages and the restrictions of the technique, a cadaveric study of supraclavicular and axillary approaches was conducted. We found that the axillary approach was useful and advantageous for lower roots, particularly for thoracic outlet syndrome (TOS). This report will focus on the evaluation of axillary robotic approach as the advantages and disadvantages of supraclavicular robotic intervention have been widely discussed in the literature.

1.1. Surgical Procedure. A human cadaver was subjected to this experiment in Paris University Ecole Europèenne de Chirurgie anatomy laboratory and da Vinci robot system was used. The cadaver was placed supine on the operating table. The left arm was tucked along the side and the right arm was placed in a semiflexed position extending toward the anesthesia location near the head, supported by foam and blankets (Figure 1). A $6 \mathrm{~cm}$ long incision was made at the right axillar line, lateral to the edge of the pectoralis major muscle (Figure 2). Blunt dissection was performed to create the working space area. A self-retaining Chung retractor was placed into the incision to elevate the pectoralis major muscle flap. The robot was docked as a camera; right and left robotic arm were adapted in the incision area (Figure 3). A $10 \mathrm{~mm} 0^{0}$ downlooking scope, Maryland forceps, and a curved scissors were introduced through the incision. The working space was maintained with the self-retaining retractor, without $\mathrm{CO}_{2}$ insufflation (Figure 4). First rib was found; C8-T1 and lower truncus were identified.

The subclavian artery was seen in front of the truncus and was positioned to the posterior of the working space. Anterior scalene muscle attachment and subclavian vein were seen anterior to the muscle. Subclavian artery was dissected from the plexus and truncus of the lower plexus was exposed with blunt dissection. The plexus was exposed thoroughly from $\mathrm{T} 1$ to C7 levels. In this surgical setting, the operating surgeon, who has a wide experience in open brachial surgery of the 


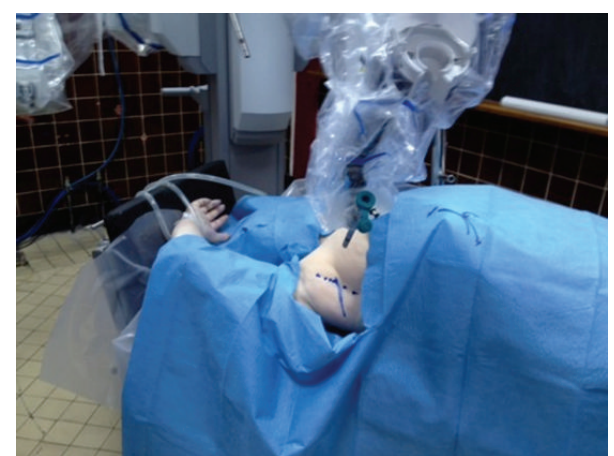

FIGURE 1: The picture showing setup position of the cadaver.

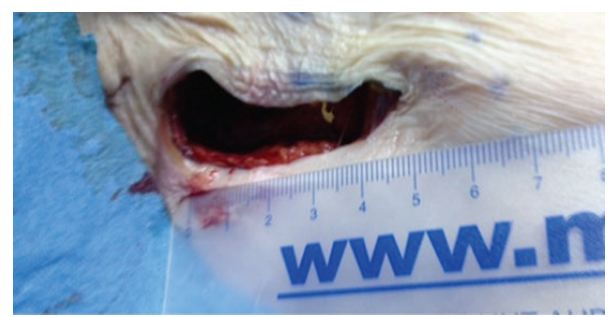

FIGURE 2: The picture showing the incision.

brachial plexus, reported that lower brachial plexus exposure was easier from the axillary working area and a more wide range of motion was achieved to manipulate the robotic tools compared to the supraclavicular exposure for lower part of the brachial plexus.

\section{Discussion}

The development of robotic-assisted minimally invasive techniques began in urology, general surgery, and gynecology because of the generally large working spaces available in the abdomen for these types of surgeries [1-4]. Since then, other surgeons have sought to use robotic devices in other areas, such as the brachial plexus $[5,6]$.

Brachial plexus dysfunction can be the result of shoulder trauma $[7,8]$. Palsy may occur with shoulder dislocation and/or traction injuries. It can also occur with TOS, which encompasses three separate disorders involving compression of the subclavian artery, subclavian vein, or brachial plexus in the triangular space bordered by the first rib, clavicle, and scalene muscles $[9,10]$. Compression of the vesselnerve package at the thoracic inlet has been treated with soft-tissue (scalene muscle) release and/or bone (first rib) resection [9]. Surgical approaches to first rib resection may be transthoracic, transaxillary, supraclavicular, infraclavicular, or thoracoscopic $[9,10]$. However, these approaches are typically associated with incomplete resection of the most medial portion of the first rib and neurovascular complications [11]. Theoretically, a minimally invasive transthoracic approach can obviate these problems, enabling complete resection of the offending portion of the first rib without

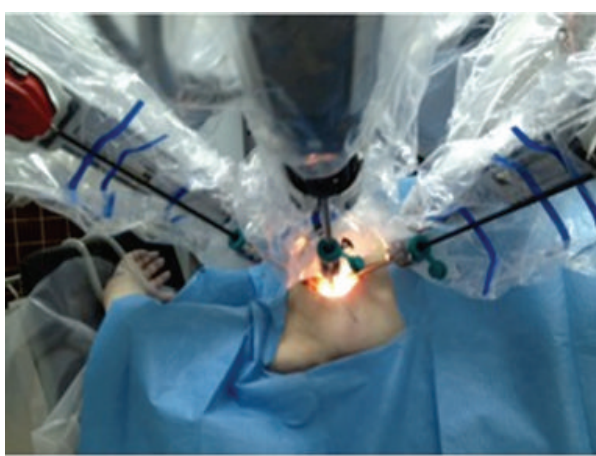

Figure 3: The picture showing the setup position of the robot.

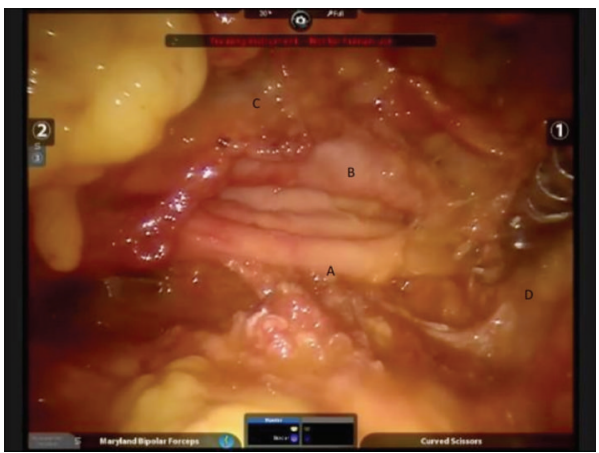

FIgURE 4: The picture showing the anatomical exposure of the lower part of the brachial plexus. A-lower truncal level, B-subclavian artery, C-subclavian vein, and D-fist rib.

neurovascular complication. Gharagozloo et al. and Martinez et al., respectively, reported successful results of robotic en bloc first rib resection for TOS treatment via transthoracic and transaxillary approaches $[9,11]$. Gharagozloo et al. and Martinez et al's techniques were only bony interventions and as being intrathoracic these need to be lung collapsed and lung complication can be waiting risk.

Open brachial plexus interventions can be performed using a supraclavicular or axillary approach. Although Liverneaux et al. reported techniques and results of upper brachial plexus injury intervention via robotic surgery with a supraclavicular approach, they described the disadvantages as a narrow working space and difficulty to expose the C7 vertebra $[1,5,12]$. To our knowledge, this report is the first to objectively describe robotic axillar brachial plexus exposure. Thus, we discuss the theoretical and clinical advantages and disadvantages of the axillary approach in the present report.

2.1. Benefits of Robotic Surgery. The development of robotassisted surgery has revealed new perspectives in peripheral nerve microsurgery. Minimally invasive robot-assisted surgery could lead to modification of the classic algorithm for the treatment of traumatic brachial plexus lesions $[6,8]$. To date, exploration of these lesions has not been attempted less than 3 months after the traumatic event because clinical 
examination cannot provide an accurate diagnosis or reliable prognosis in these first weeks [13]. Early intervention may enable initial assessment of the lesion and repair of potentially graftable nerve roots. Several robotics properties are particularly adapted to microsurgery, such as highresolution three-dimensional $(3 \mathrm{D})$ visualization with up to $\times 40$ magnification, up to 10 -fold magnification of surgical movements, elimination of physiological tremors, and the provision of ergonomic work conditions for otherwise uncomfortable surgery. Robotic surgical systems allow highdefinition magnified 3D visualization of the operative field, provide significant instrument maneuverability, even within a confined space, and may overcome the shortcomings of conventional approaches $[2,5]$.

Axillary (infraclavicular) brachial plexus intervention via robotic surgery has not been described previously. Axillary intervention was previously performed as an open procedure to expose the plexus or resect the first rib for the treatment of TOS [9]. Martinez et al. described first rib resection via robotic surgery but not to address plexus injury without transthoracic exposure, a novel minimally invasive approach to the first rib from inside of the chest [9]. In addition, Gharagozloo et al. reported first rib resection via transthoracic robotic surgery for Paget-Schroetter disease [11]. Martinez et al's techniques were considered more useful for lower brachial plexus viewing and assessing according to Gharagozloo et al.s but these two techniques describe only bony interventions.

2.2. Disadvantages of Robotic Surgery. The experience of the whole surgical team with robotic technology is important for the procedure. During learning curve period, two staff surgeons are required to participate in all procedures to ensure the safety of the program [5,9]. Martinez et al. reported importance of the learning curve, not only for the surgeon but also for the entire surgical team and 180 minutes for the initial 10 cases [9].

A second problem associated with robotic surgery is patient selection [1]. Reported exclusion criteria include a history of previous incision in the same area and obesity, which present difficulties in robotic surgery initiation. We agree with these criteria.

Other drawbacks of this new surgical approach are the increased cost of surgical equipment and longer operating time, especially during the learning curve period. However, we believe that the avoidance of a classic incision leads to significant patient satisfaction for cosmetic reasons and we believe that demand for this procedure from a select group of patients justifies the exploration of alternative ways to avoid classic brachial plexus exposure.

\section{Conclusion}

This report presents our initial experience with robot-assisted axillary exposure of the brachial plexus region. In our opinion robotic surgery will be used routinely in the future for brachial plexus surgery and particularly for TOS that is caused by bone and/or soft tissue. However, newer dedicated surgical instruments need to be developed and further studies should be conducted to evaluate in vivo application and results of this novel approach.

\section{Conflict of Interests}

All named authors hereby declare that they have no conflict of interests to disclose. This research received no specific grant from any funding agency in the public, commercial, or notfor-profit sectors.

\section{References}

[1] P. A. Liverneaux and G. R. Mantovani, Brachial Plexus: Use of the Da Vinci Robot, Thieme, New York, NY, USA, 2013.

[2] B. Bush, L. W. Nifong, and W. R. Chitwood Jr., "Robotics in cardiac surgery: past, present, and future," Rambam Maimonides Medical Journal, vol. 4, no. 3, Article ID e0017, 2013.

[3] N. C. Buchs, F. Pugin, F. Ris, F. Volonte, P. Morel, and B. Roche, "Early experience with robotic rectopexy," International Journal of Medical Robotics and Computer Assisted Surgery, vol. 9, no. 4, pp. e61-e65, 2013.

[4] C. A. Giedelman, H. Abdul-Muhsin, O. Schatloff et al., "The impact of robotic surgery in urology," Actas Urológicas Españolas, vol. 37, no. 10, pp. 652-657, 2013.

[5] P. Liverneaux, E. Nectoux, and C. Taleb, "The future of robotics in hand surgery," Chirurgie de la Main, vol. 28, no. 5, pp. 278285, 2009.

[6] E. Nectoux, C. Taleb, and P. Liverneaux, "Nerve repair in telemicrosurgery: an experimental study," Journal of Reconstructive Microsurgery, vol. 25, no. 4, pp. 261-265, 2009.

[7] J. C. Garcia Jr., F. Lebailly, G. Mantovani et al., "Telerobotic manipulation of the brachial plexus," Journal of Reconstructive Microsurgery, vol. 28, no. 7, pp. 491-494, 2012.

[8] G. Mantovani, P. Liverneaux, J. C. Garcia Jr., S. H. Berner, M. S. Bednar, and C. J. Mohr, "Endoscopic exploration and repair of brachial plexus with telerobotic manipulation: a cadaver trial," Journal of Neurosurgery, vol. 115, no. 3, pp. 659-664, 2011.

[9] B. D. Martinez, C. S. Wiegand, P. Evans, A. Gerhardinger, and J. Mendez, "Computer-assisted instrumentation during endoscopic transaxillary first rib resection for thoracic outlet syndrome: a safe alternate approach," Vascular, vol. 13, no. 6, pp. 327-335, 2005.

[10] A. W. Nichols, "Diagnosis and management of thoracic outlet syndrome," Current Sports Medicine Reports, vol. 8, no. 5, pp. 240-249, 2009.

[11] F. Gharagozloo, M. Meyer, B. J. Tempesta et al., "Robotic en bloc first-rib resection for Paget-Schroetter disease, a form of thoracic outlet syndrome: technique and initial results," Innovations, vol. 7, no. 1, pp. 39-44, 2012.

[12] P. A. Liverneaux, L. C. Diaz, J.-Y. Beaulieu, S. Durand, and C. Oberlin, "Preliminary results of double nerve transfer to restore elbow flexion in upper type brachial plexus palsies," Plastic \& Reconstructive Surgery, vol. 117, no. 3, pp. 915-919, 2006.

[13] J. Braga-Silva, D. Gehlen, and C. R. M. Kuyven, "Endoscopic exploration of a brachial plexus injury," Journal of Reconstructive Microsurgery, vol. 22, no. 7, pp. 539-541, 2006. 


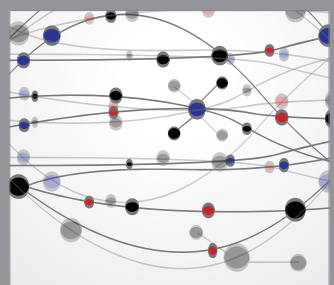

The Scientific World Journal
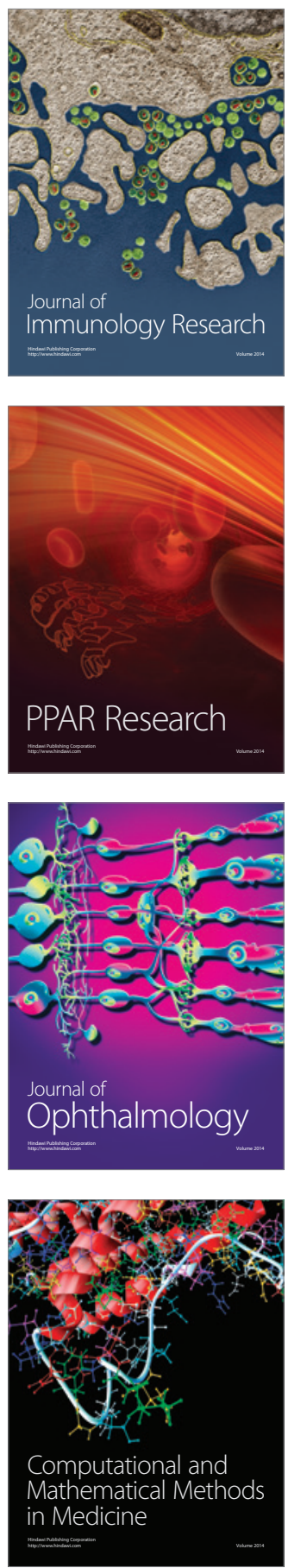

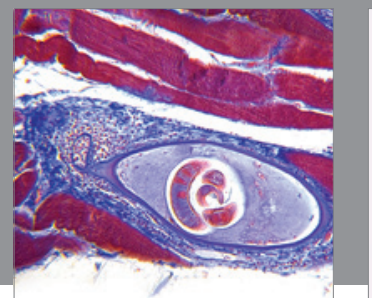

Gastroenterology

Research and Practice
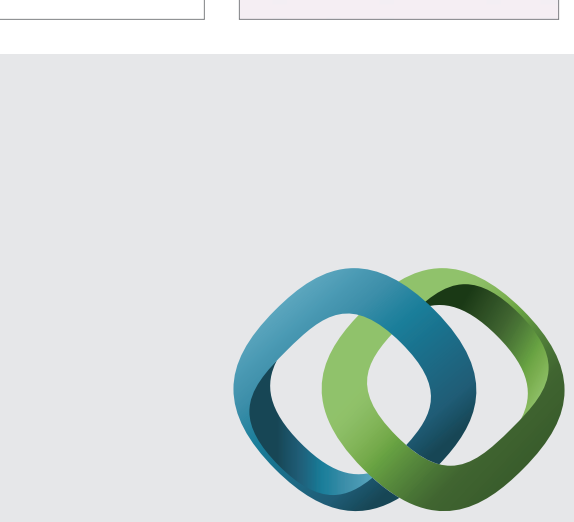

\section{Hindawi}

Submit your manuscripts at

http://www.hindawi.com
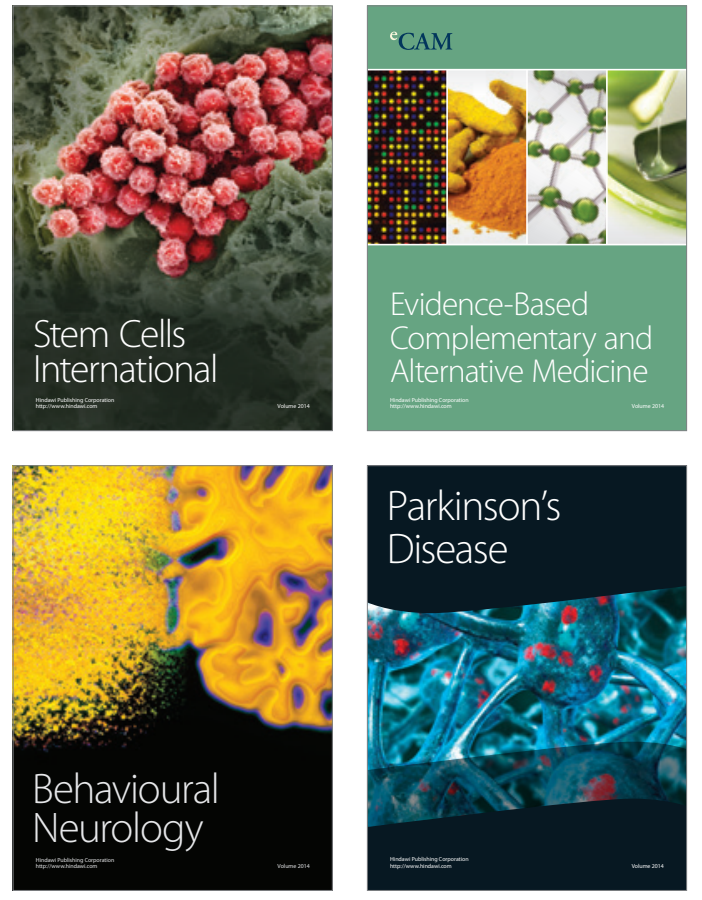
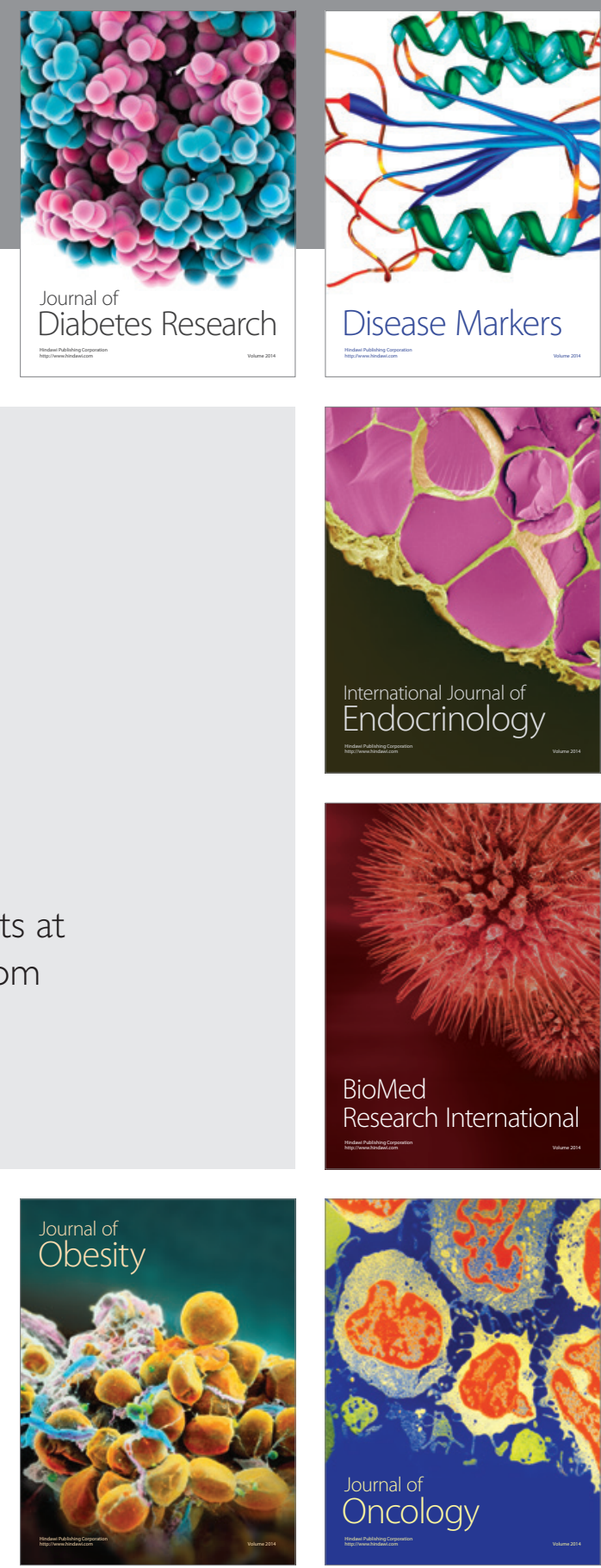

Disease Markers
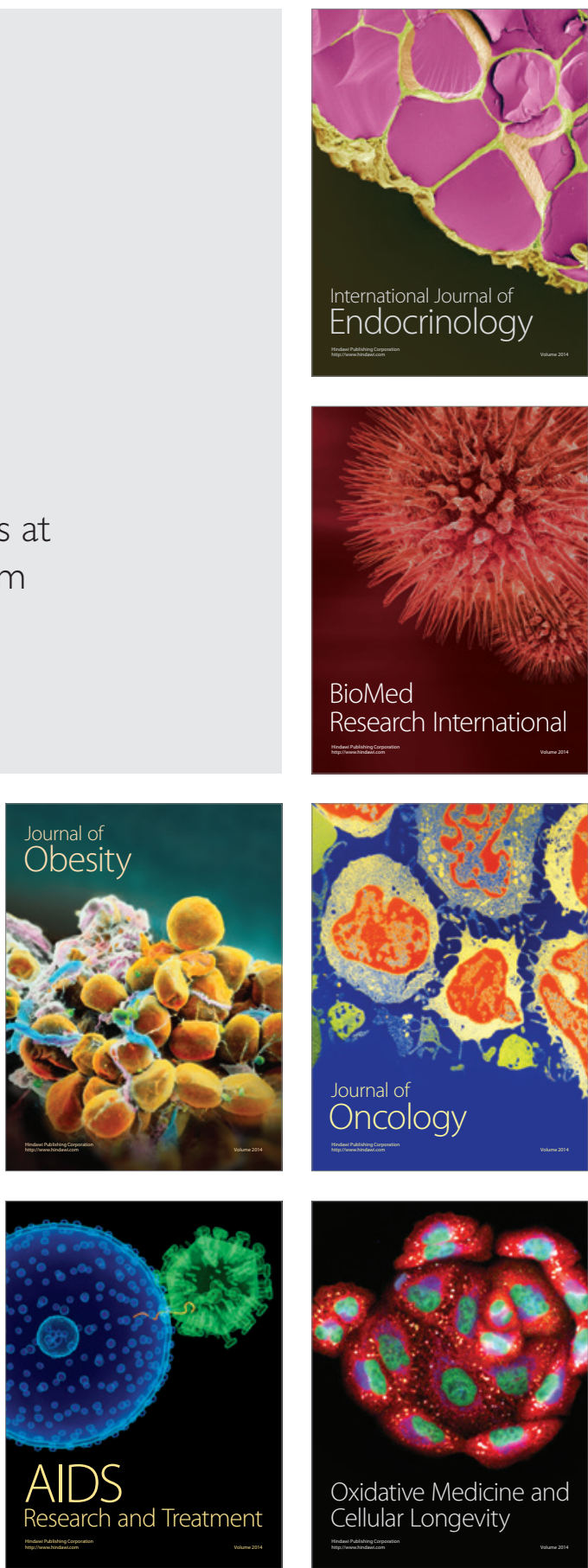